\title{
Optimizing Column Length and Particle Size in Preparative Batch Chromatography Using Enantiomeric Separations of Omeprazole and Etiracetam as Models: Feasibility of Taguchi Empirical Optimization
}

\author{
Jörgen Samuelsson ${ }^{1}$ (D - Marek Leśko ${ }^{2} \cdot$ Martin Enmark $^{1} \cdot$ Joakim Högblom $^{3} \cdot$ Anders Karlsson $^{4}$. \\ Krzysztof Kaczmarski
}

Received: 23 January 2018 / Revised: 26 March 2018 / Accepted: 5 April 2018 / Published online: 25 April 2018

(c) The Author(s) 2018

\begin{abstract}
The overreaching purpose of this study is to evaluate new approaches for determining the optimal operational and column conditions in chromatography laboratories, i.e., how best to select a packing material of proper particle size and how to determine the proper length of the column bed after selecting particle size. As model compounds, we chose two chiral drugs for preparative separation: omeprazole and etiracetam. In each case, two maximum allowed pressure drops were assumed: 80 and 200 bar. The processes were numerically optimized (mechanistic modeling) with a general rate model using a global optimization method. The numerical predictions were experimentally verified at both analytical and pilot scales. The lower allowed pressure drop represents the use of standard equipment, while the higher allowed drop represents more modern equipment. For both compounds, maximum productivity was achieved using short columns packed with small-particle size packing materials. Increasing the allowed backpressure in the separation leads to an increased productivity and reduced solvent consumption. As advanced numerical calculations might not be available in the laboratory, we also investigated a statistically based approach, i.e., the Taguchi method (empirical modeling), for finding the optimal decision variables and compared it with advanced mechanistic modeling. The Taguchi method predicted that shorter columns packed with smaller particles would be preferred over longer columns packed with larger particles. We conclude that the simpler optimization tool, i.e., the Taguchi method, can be used to obtain "good enough" preparative separations, though for accurate processes, optimization, and to determine optimal operational conditions, classical numerical optimization is still necessary.
\end{abstract}

Keywords Preparative chromatography $\cdot$ Omeprazole $\cdot$ Etiracetam $\cdot$ Optimization of productivity $\cdot$ Taguchi optimization $\cdot$ Equilibrium-dispersive model

Electronic supplementary material The online version of this article (https://doi.org/10.1007/s10337-018-3519-z) contains supplementary material, which is available to authorized users.

Jörgen Samuelsson

jorgen.samuelsson@kau.se

$\bowtie$ Krzysztof Kaczmarski

kkaczmarski@prz.edu.pl

1 Department of Engineering and Chemical Sciences, Karlstad University, 65188 Karlstad, Sweden

2 Department of Chemical Engineering, Rzeszow University of Technology, 35959 Rzeszow, Poland

3 Akzo Nobel Pulp and Performance Chemicals AB, 44580 Bohus, Sweden

4 AstraZeneca R\&D, 43183 Mölndal, Sweden

\section{Introduction}

Preparative chromatography is extensively used for peptides, bio separations, chiral separations, as well as the new biosimilar drugs such as mRNA and oligonucleotides [1-7]. Batch-mode process chromatography is the best generic method to obtain pure drug/drug candidate components in amounts under $10 \mathrm{~kg}$ in the discovery stage of pharmaceutical development. The process is mainly carried out using empirical optimization methods, such as touching-band separation, but also using numerical optimization based on chromatographic models of varying complexity [6, 8-14]. It is of highest importance for both preparative and analytical chromatography to take a scientific step from using 
empirical data and instead use powerful modeling to secure generation of scientific and mechanistic understanding (predictive science). This is well in line with the upcoming and for the pharmaceutical industry important ICHQ12 guideline [15]. There are still no strict criteria for successful preparative separation, despite some longstanding rules of thumb, such as having a retention factor as small as possible for the first-eluted component collected and dissolving the sample in a concentration close to its solubility in a particular eluent $[6,16]$.

One recent numerical study based on the chiral resolution of racemic omeprazole on an amylose tris (3,5-dimethyl phenyl carbamate)-coated macroporous silica column investigated how maximum productivity depends on the maximum allowed pressure drop and on the packing material particle size for fixed-size analytical columns [17]. It was found that the optimum particle size was large for separations conducted at low pressures, but that at higher pressures, 10- and 5- $\mu \mathrm{m}$ particles were more productive. A later study used Monte Carlo simulations of 1000 randomly selected separation systems to draw more general conclusions [16]. It was found that it is almost always beneficial to use shorter columns with higher pressure drops. Moreover, the dependence of productivity on packing particle size, as mentioned above, was verified and can be summarized as follows: (1) if the pump's maximum flow rate is the limiting factor, use smaller particle-size packing; but (2) if the system pressure is the limiting factor, use larger-particle-size $(\leq 40 \mu \mathrm{m})$ packing [16-18].

The model compounds in this study are the proton-pump inhibitor omeprazole and the antiepileptic levetiracetam (an enantiomer of etiracetam). Racemic omeprazole and its biologically more potent $S$-enantiomer (esomeprazole) are synthesized without using chromatography [19]. Omeprazole was selected, because it has previously been studied from the perspective of chromatographic separation and optimization $[3,8,17]$. Levetiracetam was selected, because it is currently produced using large-scale continuous chromatography [6] and it was the first active pharmaceutical ingredient (API) produced this way [20]. An integrated synthesis and chromatographic process for resolving levetiracetam from etiracetam was designed by UCB in the 1990s and 2000s [6], and chromatography was found to be the most economic method. Smaller particles were deemed uneconomic due to the higher cost of the material and higher required pressures in the chromatographic system [3].

The aim of this investigation is to determine how the Dynamic Axial Compression (DAC) column should be packed (i.e., determine the appropriate column length and stationary-phase particle size) to achieve optimal productivity. For over 20 years, preparative batch LC has been dominated by the DAC mode, in which a hydraulically actuated piston allows any column length to be selected, while the column diameter is fixed [21]. The process is first optimized using numerical modeling, but, since numerical optimization is tedious from a practical perspective, a simpler statistical optimization approach, i.e., the Taguchi method, is evaluated as an alternative. The Taguchi method exploits special standard orthogonal arrays, which has been successfully applied in many manufacturing industries and experimental designs [22], and it is well suited for discrete variables. Experimentally, the two separation processes were performed at maximum allowed backpressures of 80 and 200 bar to determine whether pressure affected the optimal column length and stationary-phase particle size in these cases.

\section{Theory}

\section{Column Model}

This study used the equilibrium-dispersive model equivalent to general rate (EDEG) model, which is based on the equilibrium-dispersive (ED) model [5, 9], described as follows:

$\frac{\partial c_{i}}{\partial t}+\frac{\left(1-\varepsilon_{t}\right)}{\varepsilon_{t}} \frac{\partial q_{i}}{\partial t}+\frac{u}{\varepsilon_{t}} \frac{\partial c_{i}}{\partial z}=\frac{\partial}{\partial z}\left(D_{a, z} \frac{\partial c_{i}}{\partial z}\right)$,

where $c_{i}$ and $q_{i}$ are the concentrations of the mobile and stationary phases, respectively, $u$ is the superficial velocity, $\varepsilon_{\mathrm{t}}$ is the total external porosity, $t$ is time, and $D_{a, z}$ is an apparent dispersion coefficient whose value can be determined from the measured height equivalent to the theoretical plate (HETP). For preparative chromatography, the column works in the nonlinear part of the isotherm curve and $D_{a, z}$ depends indirectly on the sample concentration [21]. When the transport-dispersive model is to be used, one must correctly calculate the effective mass transfer coefficient, which also depends on concentration [23]. In the present work, we analyzed a preparative process for species separation and decided to apply a version of the EDEG model proposed by Antos et al. [21] and Kaczmarski et al. [23, 24].

In solving Eq. (1), the apparent dispersion coefficient is calculated from the following relationship, for the first and second components:

$D_{a, z}=\frac{D_{\mathrm{L}} \varepsilon_{\mathrm{e}}}{\varepsilon_{\mathrm{t}}}+\left(\frac{k_{1}}{1+k_{1}}\right)^{2} \frac{u^{2} d_{\mathrm{p}}}{\varepsilon_{\mathrm{t}} \varepsilon_{\mathrm{e}} F_{\mathrm{e}} 6}\left[\frac{d_{\mathrm{p}}}{10 D_{\text {eff }}}+\frac{1}{k_{\mathrm{ext}}}\right]$,

where $\varepsilon_{\mathrm{e}}$ is external porosity, $D_{\mathrm{L}}$ is an axial dispersion coefficient, and $k_{1}, F_{\mathrm{e}}$, and $D_{\text {eff }}$ can be expressed as:

$k_{1}=F_{\mathrm{e}}\left(\varepsilon_{\mathrm{p}}+\left(1-\varepsilon_{\mathrm{p}}\right) \frac{\partial q}{\partial c}\right), \quad F_{\mathrm{e}}=\frac{1-\varepsilon_{\mathrm{e}}}{\varepsilon_{\mathrm{e}}}, \quad$ and $\quad D_{\text {eff }}=\frac{\varepsilon_{\mathrm{p}} D_{\mathrm{m}}}{\tau}$,

respectively, where $D_{\mathrm{m}}$ is diffusivity, $k_{\mathrm{ext}}$ is a mass transfer coefficient, $d_{\mathrm{p}}$ is particle diameter, $\tau$ is tortuosity, $\varepsilon_{\mathrm{p}}$ is pore 
porosity, and $\partial q / \partial c$ is approximated using the slope at $C=0$. When calculating $D_{\text {eff }}$, it is assumed that the surface diffusion can be ignored. The external porosity, $\varepsilon_{\mathrm{e}}$, is assumed to be 0.4 . The tortuosity coefficient was obtained from the following relationship:

$\tau=\frac{\left(2-\varepsilon_{\mathrm{p}}\right)^{2}}{\varepsilon_{\mathrm{p}}}$.

In this study, a bi-Langmuir-like adsorption-desorption kinetic model was used for the enantiomeric separation of omeprazole; it can be described as:

$\frac{\partial q_{i}^{j}}{\partial t}=k_{\mathrm{a}, i}^{j} C_{i}\left(q_{\mathrm{s}, i}^{j}-q_{1}^{j}-q_{2}^{j}\right)-k_{\mathrm{d}, i}^{j} q_{i}^{j}$,

where $k_{\mathrm{a}}$ and $k_{\mathrm{d}}$ are the rate constants for adsorption and desorption, respectively, $q_{\mathrm{s}}$ is the monolayer saturation capacity, and $q_{i}^{j}$ is the amount of component $i$ adsorbed on site $j$. The total amount of adsorbed compound is:

$q_{i}=q_{i}^{1}+q_{i}^{2}$,

where $q_{i}$ is the adsorbed amount of component $i$ and the superscript indicates the first site or second site.

For the enantiomeric separation of etiracetam, the following competitive bi-Langmuir isotherm model was applied:

$q_{i}=\frac{q_{\mathrm{ns}} K_{\mathrm{ns}} C_{i}}{1+K_{\mathrm{ns}}\left(C_{1}+C_{2}\right)}+\frac{q_{\mathrm{es}} K_{\mathrm{es}} C_{i}}{1+K_{\mathrm{es}, 1} C_{1}+K_{\mathrm{es}, 2} C_{2}}$,

where $q_{\mathrm{ns}}$ and $q_{\mathrm{es}}$ are the saturation capacities and $K_{\mathrm{ns}}$ and $K_{\text {es }}$ are the association equilibrium constants of the nonselective and selective adsorption sites, respectively. This isotherm model assumes two types of adsorption sites: the first type (first term) behaves identically toward the two enantiomers, while the second type (second term) is enantioselective and responsible for chiral separation. The model has been widely and successfully applied to describe adsorption isotherms of chiral solutes; for example, the 1-indanol enantiomers [25]. The benefits of using bi-Langmuir adsorption model are that it has a foundation in the physiochemical adsorption process, but a drawback is that it contains more parameters, thus making the numerical determination more complex.

The discussed model was solved with typical initial and boundary conditions and the kinetic parameters were calculated as follows. The mass transfer coefficient, $k_{\text {ext }}$, was evaluated from the Wilson-Geankoplis correlation [26]:

$S h=\frac{1.09}{\varepsilon_{\mathrm{e}}} R e^{1 / 3} S c^{1 / 3}$,

where $S h$ is the Sherwood number, Re the Reynolds number, and $S c$ the Schmidt number:
$\mathrm{Sh}=\frac{k_{\mathrm{ext}} d_{\mathrm{p}}}{D_{\mathrm{m}}}, \operatorname{Re}=\frac{u \rho d_{\mathrm{p}}}{\eta}$ and $\mathrm{Sc}=\frac{\eta}{\rho D_{\mathrm{m}}}$.

The diffusivity, $D_{\mathrm{m}}\left(\mathrm{m}^{2} \mathrm{~s}^{-1}\right)$, was calculated from the Wilke-Chang model [27]:

$D_{\mathrm{m}}=1.17 \times 10^{-16} T \frac{\left(\alpha \cdot M_{\mathrm{w}}\right)^{0.5}}{\eta \cdot V_{i}^{0.6}}$,

where $T$ is temperature $(\mathrm{K}), M_{\mathrm{w}}$ is the solute molar mass $\left(\mathrm{kg} \mathrm{mol}^{-1}\right), \eta$ is viscosity $(\mathrm{Pa} \times \mathrm{s}), \alpha=1.9$ for methanol and 1 for $60 / 40$ ethanol/heptane, $V_{i}$ is the molar volume in the mobile phase $\left(\mathrm{m}^{3} \mathrm{~mol}^{-1}\right)$, and $V_{i}=368.5 \times 10^{-3}\left(\mathrm{~m}^{3} \mathrm{kmol}^{-1}\right)$ for omeprazole and $194.5 \times 10^{-3}\left(\mathrm{~m}^{3} \mathrm{~mol}^{-1}\right)$ for etiracetam. Finally, the axial dispersion coefficient is obtained from the following relationship:

$D_{\mathrm{L}}=0.7 D_{\mathrm{m}}+0.5 u d_{\mathrm{p}}$.

In this study, the adsorption isotherm was estimated using the inverse method [28, 29], solved using orthogonal collocation on finite elements [24]. The chosen base experimental concentration profiles were estimated by minimizing the sum of squared differences between the experimental and calculated elution profiles.

\section{Optimization}

As objective function in the optimization, the productivity (Pr) (defined as gram product collected per minute), was used. In this study, the optimization problem comes down to the following expression:

$\operatorname{Max}\left(\operatorname{Pr}_{i}\right)=f\left(u, t_{\mathrm{inj}}, d_{\mathrm{p}}, L\right)$.

As the optimization problem expressed by Eq. (12) was quite complex, the hybrid method of simulated annealing coupled with a simplex algorithm was applied [30]. This hybrid optimization tool, which combines the stochastic method of simulated annealing with a deterministic simplex algorithm, is suitable for solving difficult optimization problems in which the desired global optimum is hidden among many local optima. The detailed algorithm and a study of the effectiveness of the applied optimization method were presented by Kaczmarski and Antos [31]. However, this numerical optimization of the chromatographic separation is time-consuming; therefore, a faster algorithm based on design of experiments (DoE) was also investigated.

The DoE method allows one to conduct just a few experiments to describe the system variance. In DoE, all factors (in this case, superficial fluid velocity, injection time, column length, and particle diameter) are studied at different levels (i.e., low, medium, and high). As the total number of required experiments increases rapidly with the number of factors, reduced design schemas are often employed. The 
Taguchi model is a special orthogonal array approach that drastically reduces the number of needed experiments [32]. In this study, four factors were considered at three levels. For such cases, Taguchi proposes the $L_{9}$ orthogonal array, which reduces a full-factor design of $81\left(3^{4}\right)$ experiments to only nine (see Tables S1-S4 in Electronic Supplementary Material).

The Taguchi optimum can be calculated as:

$$
(\operatorname{Pr} / L)_{\mathrm{opt}}=\bar{T}+\left(\bar{A}_{\max }-\bar{T}\right)+\left(\bar{B}_{\max }-\bar{T}\right)+\left(\bar{C}_{\max }-\bar{T}\right)+\left(\bar{D}_{\max }-\bar{T}\right),
$$

where $\bar{A}_{\max }, \bar{B}_{\max }, \bar{C}_{\max }$ and $\bar{D}_{\max }$ are the highest average effects of the factors corresponding to the optimal values of the factors; and $\bar{T}$ is the grand average of productivity, obtained by averaging the results of all trial combinations of factors and levels:

$\bar{T}=\frac{1}{9} \sum_{i=1}^{9}\left(\operatorname{Pr}_{i} / L\right)$

where $\operatorname{Pr}_{i}$ is the productivity of individual trials. The average effect of a factor at a given level is calculated by averaging all productivities containing the factor level of interest:

$$
\begin{array}{r}
\overline{A_{i}}=\frac{1}{3} \sum_{j=1}^{3}\left(\operatorname{Pr}_{i, j} / L\right), \quad \overline{B_{i}}=\frac{1}{3} \sum_{j=1}^{3}\left(\operatorname{Pr}_{i, j} / L\right), \\
\overline{C_{i}}=\frac{1}{3} \sum_{j=1}^{3}\left(\operatorname{Pr}_{i, j} / L\right) \quad \text { and } \overline{D_{i}}=\frac{1}{3} \sum_{j=1}^{3}\left(\operatorname{Pr}_{i, j} / L\right),
\end{array}
$$

where $\bar{A}_{i}, \bar{B}_{i}, \bar{C}_{i}$ and $\bar{D}_{i}$ are the average effects (i.e., productivity) of the considered factors at the $i$ th level; and $\operatorname{Pr}_{i, j}$ is the productivity calculated for the considered factor at the $i$ th level in accordance with the $L_{9}$ array (see Tables S1-S4).

\section{Experimental}

\section{Apparatus and Chemicals}

The solutes used in this study were $R / S$-omeprazole and $R / S$ etiracetam obtained from AstraZeneca (Mölndal, Sweden) and UCB Pharma (Bruxelles, Belgium), respectively. As eluent, HPLC-grade (99.99\%) methanol from Fisher Scientific (Loughborough, UK) was used for omeprazole [17] and 60/40\% (v/v) ethanol/heptane was used for etiracetam [33]. The ethanol was analytical grade (99.90\%) from VWR International (Fontenay-sous-Bois, France) and the heptane was HPLC grade (99\%) from Fisher Scientific (Loughborough, UK). In all experiments, 1,3,5-tri-tert-butylbenzene
(97\%) from Sigma-Aldrich (Steinheim, Germany) was used to estimate the void volume. The analytical scale experiments were performed on Agilent 1100 and 1200 HPLC systems (Palo Alto, CA, USA) consisting of a binary pump, a preparative auto-sampler $(900 \mu \mathrm{L}, \max .200$ bar $)$ and a diode array UV detector. At analytical scale, 0.46-cm-i.d. Kromasil AmyCoat columns from Akzo Nobel Pulp and Performance Chemicals AB (Bohus, Sweden), 10-, 15-, and $25-\mathrm{cm}$ long and packed with $10-$ and $25-\mu \mathrm{m}$ particles, were used. An additional $10 \times 0.46-\mathrm{cm}$ AmyCoat column packed with $5-\mu \mathrm{m}$ particles was used in the validation experiments. The column temperature was kept at $23.0^{\circ} \mathrm{C}$ by immersing the columns in a temperature-controlled water bath. The pilot-scale experiments were performed using a Packer LC50.340 VE100 PS TH column (Novasep, Boothwyn, PA, USA), 50-mm i.d., packed to a bed height of $105 \mathrm{~mm}$ with AmyCoat 5- $\mu \mathrm{m}$ particles (same batch as used in the analytical columns), together with two K-1800 preparative pumps and a K-2600 UV detector (Knauer, Berlin, Germany).

\section{Procedures}

The holdup volumes were estimated on all columns for both eluents by injecting $5 \mu \mathrm{L}$ of diluted 1,3,5-tri-tert-butylbenzene three times at a flow rate of $2 \mathrm{~mL} \mathrm{~min}{ }^{-1}$ (except at pilot scale, at which the flow rate was $205 \mathrm{~mL} \mathrm{~min}^{-1}$ ) when detecting at $220 \mathrm{~nm}$. The average elution volume was considered to be the void volume of the column. Analytical injections of $5 \mu \mathrm{L}$ of $1 \mathrm{~g} \mathrm{~L}^{-1}$ omeprazole dissolved in $\mathrm{MeOH}$ and of $0.35 \mathrm{~g} \mathrm{~L}^{-1}$ etiracetam dissolved in 60/40\% (v/v) ethanol/ heptane were performed and recorded at $220 \mathrm{~nm}$. The pressure/flow rate dependence was determined at $1-5 \mathrm{~mL} \mathrm{~min}^{-1}$ for all column lengths and packing material particle sizes using pure methanol as well as 60/40\% (v/v) ethanol/heptane as the eluent. The system pressure contributions of the Agilent 1100 and 1200 systems were also measured without columns at $1-5 \mathrm{~mL} \mathrm{~min}{ }^{-1}$ using both eluents. Overloaded duplicate samples of 50,100, 200, 400, 600, and $900 \mu \mathrm{L}$ of $30 \mathrm{~g} \mathrm{~L}^{-1}$ omeprazole and triplicate samples of 50,75, $100,150,200,250,300$, and $350 \mu \mathrm{L}$ of $60 \mathrm{~g} \mathrm{~L}^{-1}$ etiracetam were injected into each column. A flow rate of $2 \mathrm{~mL} \mathrm{~min}^{-1}$ was maintained for both substances. Chromatograms were recorded at $345 \mathrm{~nm}$ for omeprazole and $260 \mathrm{~nm}$ for etiracetam.

\section{Calculations}

The adsorption parameters needed to solve Eq. (1) were estimated from the experimental overloaded elution profiles using the inverse method. Before calculating the adsorption parameters using the inverse method, the UV absorbance was converted to concentration. This was done by fitting 
the UV response to the elution profile and requiring that the mass balance equation be fulfilled [34].

The optimum productivity was calculated as a function of the flow rate, column length, packing material diameter, and injection time. As constraints, the maximum allowed backpressure was set to 80 or 200 bar, and the purity of the products to $99 \%$.

In the calculations, the particle size was varied between 5 and $25 \mu \mathrm{m}$, the flow rate was set to $5 \mathrm{~mL} \mathrm{~min}^{-1}$, the pressure constraint was as calculated from the experimental measurements, the column length was varied between 100 and $250 \mathrm{~mm}$, and the injection volume was limited to a maximum of $900 \mu \mathrm{L}$. All calculations were conducted assuming the column to have an inner diameter of $4.6 \mathrm{~mm}$. The particle diameter, superficial fluid velocity, and column length were limited by the pressure drop, which is the sum of the column pressure drop and the pressure drop contributed by the system. The pressure drop of the Agilent system was approximated by a polynomial relationship based on experimental data and the column pressure drop was calculated using the Blake-Kozeny equation.

In the numerical optimization, the particle diameter and column length were considered continuous variables in the optimization, so they were rounded to the nearest real value after optimization.

In the Taguchi approach, four variables (factors) were considered at three levels, as follows: superficial velocity of the mobile phase $\left(u_{\max }, 2 / 3 u_{\max }\right.$, and $\left.1 / 3 u_{\max }\right)$, injection volume $\left(0.9,0.6\right.$, and $\left.0.3 \mathrm{~cm}^{3}\right)$, column length $(25,15$, and $10 \mathrm{~cm})$, and particle diameter $(25,10$, and $5 \mu \mathrm{m})$. The $u_{\max }$ is dependent on the eluent and the maximum allowed pressure drop ( 80 or 200 bar) in the separation.

\section{Results and Discussion}

\section{Numerical Model Validation}

Previously, the equilibrium-dispersive (ED) column model has been used to describe the enantiomeric separation of omeprazole [17]. However, the analytical peaks for omeprazole were tailing. To deduce whether this peak tailing is due to thermodynamic (overloading) reasons, the column load of omeprazole was reduced from $25 \mu \mathrm{g}$ to $1.25 \mu \mathrm{g}$ on a $250 \times 4.6 \mathrm{~mm}$ column without any reduction in peak tailing, see Fig. S1 in the Electronic Supplementary Material. We also noted that increasing the flow rate from 0.25 to $2 \mathrm{~mL} \mathrm{~min}{ }^{-1}$ resulted in increased tailing, see Fig. S2 in the Electronic Supplementary Material. We, therefore, conclude that the peak tailing occurs due to kinetic reasons, namely, a slow adsorption-desorption process. From a model perspective, the kinetic tailing is handled using a kinetic representation of the adsorption isotherm, see Eq. (5).

To calculate the elution profiles more accurately, the EDEG model was used (see subsection "Column Model" in the "Theory" section). The inverse method was used to estimate the adsorption isotherm parameters for the enantiomeric separation of omeprazole and etiracetam at different column lengths and particle sizes. Figure 1
Fig. 1 Experimental chromatograms (blue lines) and model predictions (red lines) of the enantiomeric separation of ome-

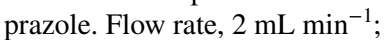
injection volume, $200 \mu \mathrm{L}$. Columns: a $10 \mu \mathrm{m}, 4.6 \times 100 \mathrm{~mm}$; b $10 \mu \mathrm{m}, 4.6 \times 150 \mathrm{~mm}$; c $10 \mu \mathrm{m}, 4.6 \times 250 \mathrm{~mm}$; d $25 \mu \mathrm{m}, 4.6 \times 100 \mathrm{~mm} ; \mathbf{e} 25 \mu \mathrm{m}$, $4.6 \times 150 \mathrm{~mm}$; and $\mathbf{f} 25 \mu \mathrm{m}$, $4.6 \times 250 \mathrm{~mm}$
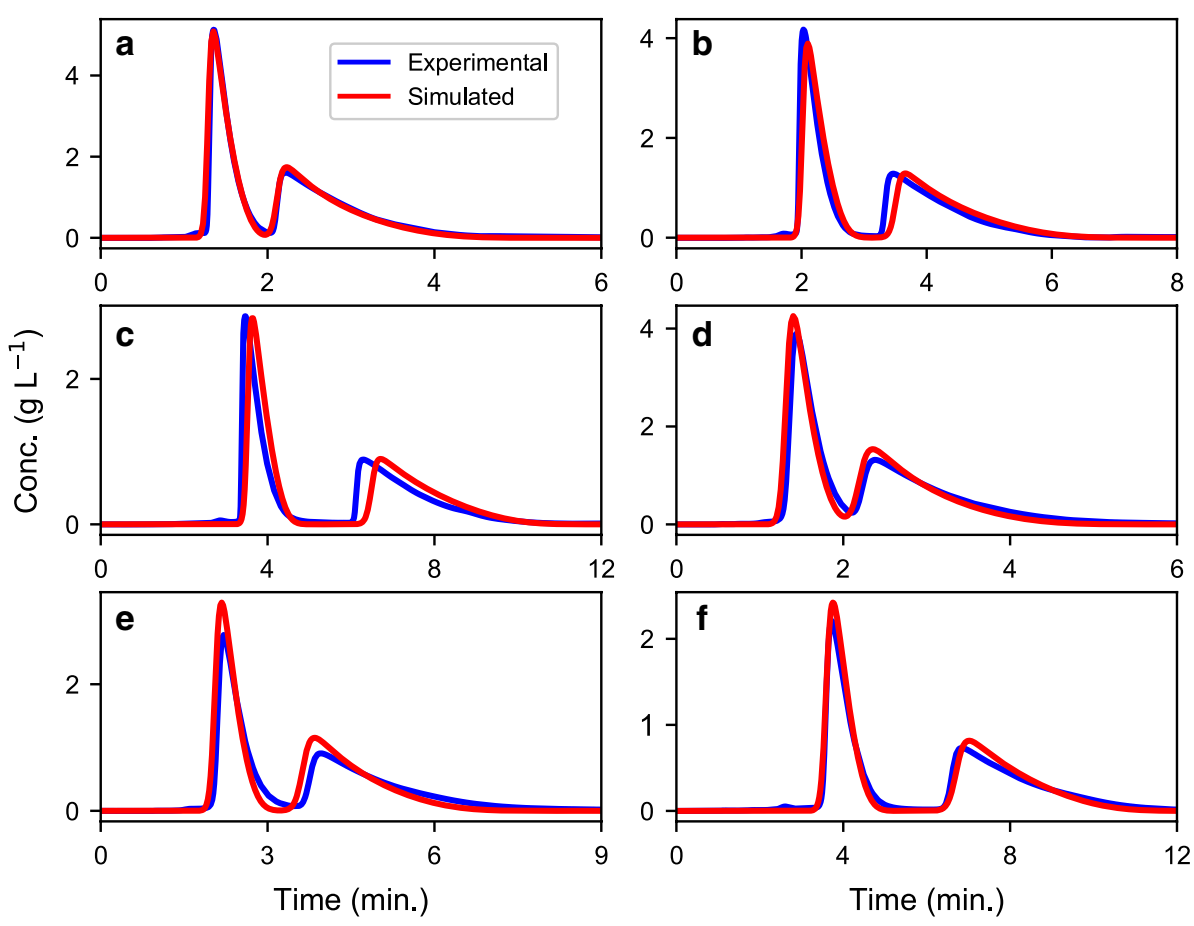


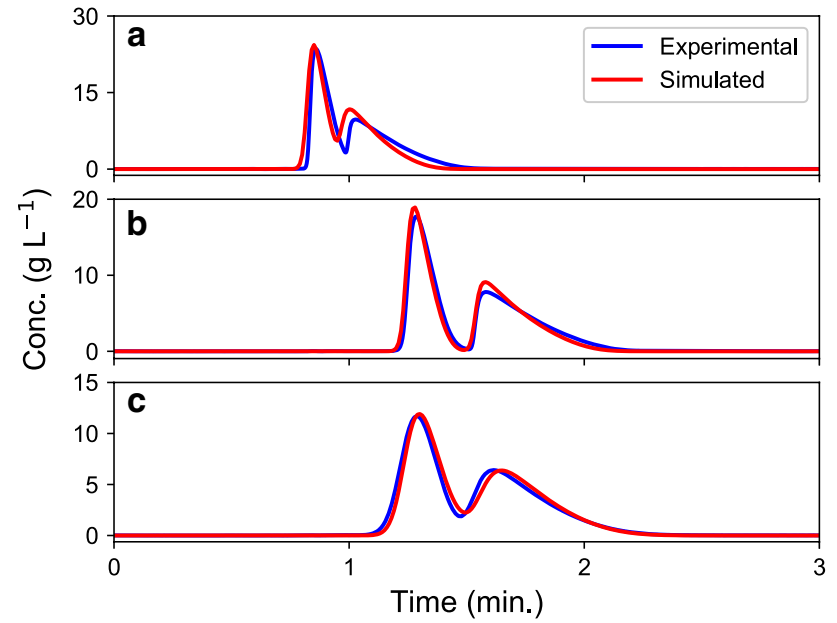

Fig. 2 Experimental chromatograms (blue lines) and model predictions (red lines) of the enantiomeric separation of etiracetam. Flow rate, $2 \mathrm{~mL} \mathrm{~min}{ }^{-1}$; injection volume, $150 \mu \mathrm{L}$. Columns: a $5 \mu \mathrm{m}$, $4.6 \times 100 \mathrm{~mm} ; \mathbf{b} 10 \mu \mathrm{m}, 4.6 \times 150 \mathrm{~mm}$; and c $25 \mu \mathrm{m}, 4.6 \times 150 \mathrm{~mm}$
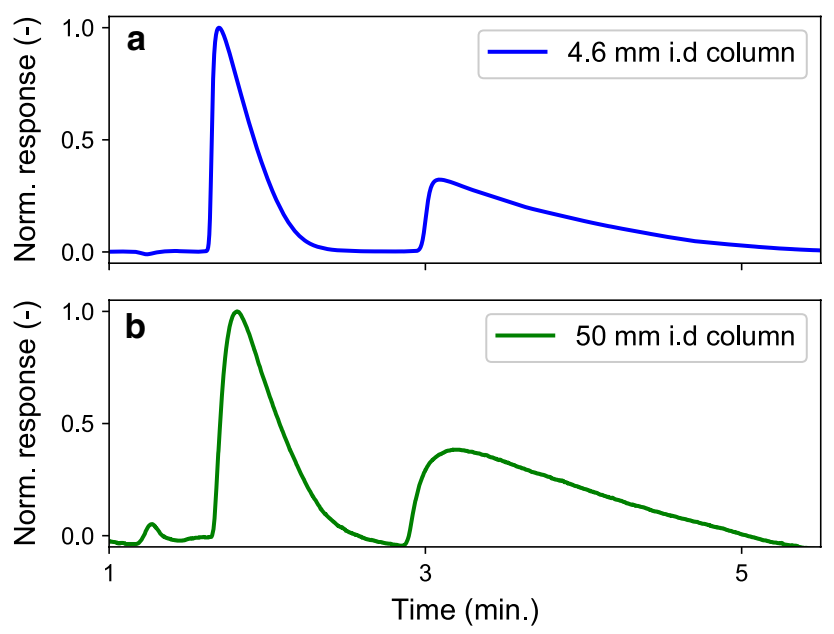

Fig. 3 Enantiomeric separation of omeprazole on AmyCoat columns at analytical scale (top, blue line) and pilot scale (bottom, green line). Analytical separation on a $100 \times 4.6 \mathrm{~mm}$ column using a $85-\mu \mathrm{L}$ sample of $30 \mathrm{~g} \mathrm{~L}^{-1}$ racemic omeprazole injected at a flow rate of $1.7 \mathrm{~mL} \mathrm{~min}{ }^{-1}$; pilot separation on a $105 \times 50 \mathrm{~mm}$ column using a 10 -mL sample of $30 \mathrm{~g} \mathrm{~L}^{-1}$ racemic omeprazole injected at a flow rate of $205 \mathrm{~mL} \mathrm{~min}^{-1}$

presents the experimental chromatograms and the model predictions for the enantiomeric separation of omeprazole, while Fig. 2 presents the corresponding experimental and predicted results for etiracetam. Inspecting Figs. 1 and 2, we can conclude that the numerical models describe the experimental data well.

To investigate potential scale-up issues, the enantiomeric separation of omeprazole conducted on a 4.6- $\mathrm{mm}$ i.d. column was compared with separation experiments conducted on a 50-mm-i.d. pilot-scale column, corresponding to a 118-times scale-up (see Fig. 3). Both columns, approximately 100-mm long, were packed with 5- $\mu \mathrm{m}$ AmyCoat packing. In Fig. 3a, the separation on the 4.6-mm-i.d. column is plotted; this separation was performed with an $85-\mu \mathrm{L}$ injection of $30 \mathrm{~g} \mathrm{~L}^{-1}$ omeprazole at a flow rate of $1.7 \mathrm{~mL} \mathrm{~min}^{-1}$. Figure $3 b$ shows the corresponding pilot-scale separation, performed with a $10-\mathrm{mL}$ injection of $30 \mathrm{~g} \mathrm{~L}^{-1}$ omeprazole at a flow rate of $205 \mathrm{~mL} \mathrm{~min}^{-1}$. As it can be seen, the experiments conducted at analytical scale and pilot scale differed only slightly in their results, so it can be concluded that this process is likely scalable and that our model could be used to predict elution profiles.

\section{Optimal Column Length and Particle Size}

Based on the model validation results, the enantiomeric separation of omeprazole and etiracetam was numerically optimized and experimentally confirmed. Table 1 presents the optimal experimental settings as well as the calculated productivity. Figures 4, 5 present the calculated optimal conditions for the enantiomeric separation of omeprazole and etiracetam at 80 and 200 bars, respectively, for the $R$ and $S$ enantiomers with overlaid experimental verification. In both cases, relatively good agreement was obtained.

The optimum particle size for omeprazole at 80 and 200 bar is $5 \mu \mathrm{m}$, and the maximum productivity was found at the minimum allowed column length of $10 \mathrm{~cm}$. When the maximum allowed pressure was increased from 80 to $200 \mathrm{bar}$, this resulted in an approximately 1.5 times higher productivity, while the solvent consumption decreased 0.6 times. The reduced solvent consumption with increased pressure is in line with the previous findings [17].

For etiracetam, the optimum packing at 80 and 200 bar is $10 \mu \mathrm{m}$ and, as in the case of omeprazole, the shortest column of $10 \mathrm{~cm}$ should be used at both pressures. Increasing the maximum allowed pressure from 80 to 200 bar led to approximately 1.4 times higher productivity, while the solvent consumption decreased by 0.6 times. This clearly indicates that pressure is the most important factor in increasing the productivity, because it allows the operational flow rate to increase. This also suggests that using smaller particles will result in more productive processes.

From Table 1, we also see, as suspected, that the productivity is always higher for the first-eluted compound (i.e., $S$-omeprazole and $R$-etiracetam), for both systems. However, the etiracetam process is approximately 4-5 times more productive than the omeprazole process, mainly because the cycle time is much shorter for 
Table 1 Optimum conditions for first- and second-eluted enantiomers of omeprazole and etiracetam, respectively

\begin{tabular}{|c|c|c|c|c|c|c|c|c|c|c|}
\hline Compound & Target & $\Delta P_{\max }$ (bar) & Method & $L(\mathrm{~cm})$ & $d_{\mathrm{p}}(\mu \mathrm{m})$ & $u(\mathrm{~cm} \mathrm{~min}-1)$ & $t_{\text {inj }}(\min )$ & $\begin{array}{l}\operatorname{Pr} \times 10^{2 \mathrm{a}} \\
\left(\mathrm{g} \min ^{-1}\right)\end{array}$ & $\begin{array}{l}\operatorname{Pr} \times 10^{2 b} \\
\left(\mathrm{~g} \min ^{-1}\right)\end{array}$ & $\begin{array}{l}\mathrm{SP} \times 10^{3} \\
\left(\mathrm{~g} \mathrm{~cm}^{-1}\right)\end{array}$ \\
\hline \multirow[t]{8}{*}{ Omeprazole } & \multirow[t]{4}{*}{ S (1) } & \multirow[t]{2}{*}{80} & $\mathrm{~T}$ & 10 & 25 & 8.32 & 0.651 & 0.8336 & 0.9376 & 0.7642 \\
\hline & & & SS & 10 & 5 & 12.20 & 0.342 & 1.337 & - & 0.8363 \\
\hline & & \multirow[t]{2}{*}{200} & $\mathrm{~T}$ & 10 & 5 & 30.08 & 0.180 & 1.678 & 1.616 & 0.4260 \\
\hline & & & SS & 10 & 5 & 29.55 & 0.119 & 1.995 & - & 0.5150 \\
\hline & \multirow[t]{4}{*}{$\mathrm{R}(2)$} & \multirow[t]{2}{*}{80} & $\mathrm{~T}$ & 10 & 5 & 8.32 & 0.651 & 0.5940 & 0.5762 & 0.5445 \\
\hline & & & SS & 10 & 5 & 12.40 & 0.186 & 0.8417 & - & 0.5177 \\
\hline & & \multirow[t]{2}{*}{200} & $\mathrm{~T}$ & 10 & 5 & 30.08 & 0.180 & 1.296 & 0.9800 & 0.3291 \\
\hline & & & SS & 10 & 5 & 29.59 & 0.1027 & 1.345 & - & 0.3469 \\
\hline \multirow[t]{8}{*}{ Etiracetam } & \multirow[t]{4}{*}{ S (2) } & \multirow[t]{2}{*}{80} & $\mathrm{~T}$ & 15 & 5 & 4.80 & 1.128 & 2.535 & 2.526 & 3.792 \\
\hline & & & SS & 10 & 10 & 12.32 & 0.087 & 4.659 & - & 2.695 \\
\hline & & \multirow[t]{2}{*}{200} & $\mathrm{~T}$ & 10 & 5 & 25.0 & 0.217 & 4.178 & 3.793 & 1.199 \\
\hline & & & SS & 10 & 10 & 29.47 & 0.040 & 6.542 & - & 1.582 \\
\hline & \multirow[t]{4}{*}{$\mathrm{R}(1)$} & \multirow[t]{2}{*}{80} & $\mathrm{~T}$ & 15 & 5 & 4.80 & 0.376 & 3.392 & 2.576 & 5.073 \\
\hline & & & SS & 10 & 10 & 12.35 & 0.140 & 6.270 & - & 3.618 \\
\hline & & \multirow[t]{2}{*}{200} & $\mathrm{~T}$ & 15 & 5 & 16.7 & 0.324 & 6.868 & 8.931 & 1.969 \\
\hline & & & SS & 10 & 10 & 23.60 & 0.062 & 7.463 & - & 2.254 \\
\hline
\end{tabular}

Two optimization methods were used: $T$ Taguchi and SS simulated annealing + simplex. The numbers 1 and 2 under "Target" indicate the firstand second-eluting enantiomers

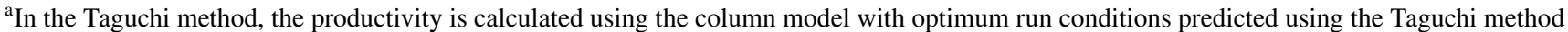

${ }^{b}$ Productivity is estimated using the Taguchi method, Eq. (13)

Fig. 4 Experimental (blue lines) and calculated (red lines) optimal conditions for the enantiomeric separation of omeprazole and etiracetam at 80 bar for the first- and second-eluted enantiomers. Optimal conditions for: a first-eluted enantiomer of omeprazole, $\mathbf{b}$ second-eluted enantiomer of omeprazole, c first-eluted enantiomer of etiracetam, and $\mathbf{d}$ second-eluted enantiomer of etiracetam
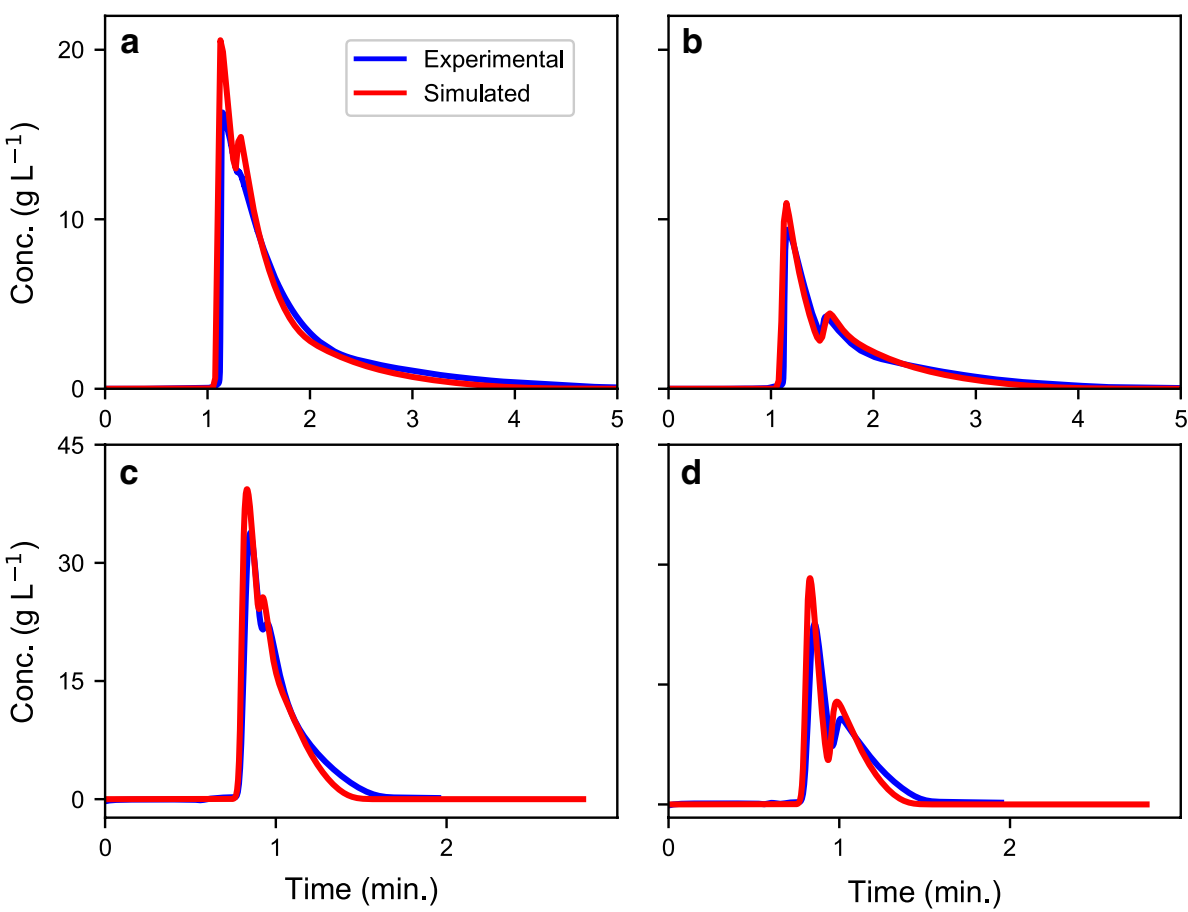

etiracetam than for omeprazole. Other factors that increase the productivity for etiracetam are that the separation system is more efficient and that the sample concentration of etiracetam is double that of omeprazole due to solubility reasons.

\section{Taguchi vs. Classical Numerical Optimization}

The Taguchi approach was investigated to see whether it could speed up the optimization process. From a practical perspective, the Taguchi method could be interesting as 
an alternative/complement to touching-band optimization, because it could simplify the selection of column length, packing material, packing material particle size, etc.

Here, the Taguchi method required only nine experiments to optimize the column length, stationary-phase packing material particle size, injection volume, as well as flow rate (see Tables S1-S4 in Electronic Supplementary Material for more information). The optimal decision variables and productivity estimated using Taguchi optimization are presented in Table 1. In Fig. 6, the normalized average effects of all decision variables are plotted for the optimization of $S$-omeprazole and $R$-etiracetam at 200 and 80 bar.
Fig. 5 Experimental (blue lines) and calculated (red lines) optimal conditions for the enantiomeric separation of omeprazole and etiracetam at 200 bar for the first- and second-eluted enantiomers. Optimal conditions for: a first-eluted enantiomer of omeprazole, b second-eluted enantiomer of omeprazole, c first-eluted enantiomer of etiracetam, and $\mathbf{d}$ second-eluted enantiomer of etiracetam

Fig. 6 Plot of factor average effect minus the grand average in Taguchi optimization, optimized system: a omeprazole $(S)$, pressure restriction 200 bar; b etiracetam $(R)$, pressure restriction 200 bar; c omeprazole $(S)$, pressure restriction 80 bar; $\mathbf{d}$ etiracetam $(R)$, pressure restriction 80 bar. The gray line is the grand average
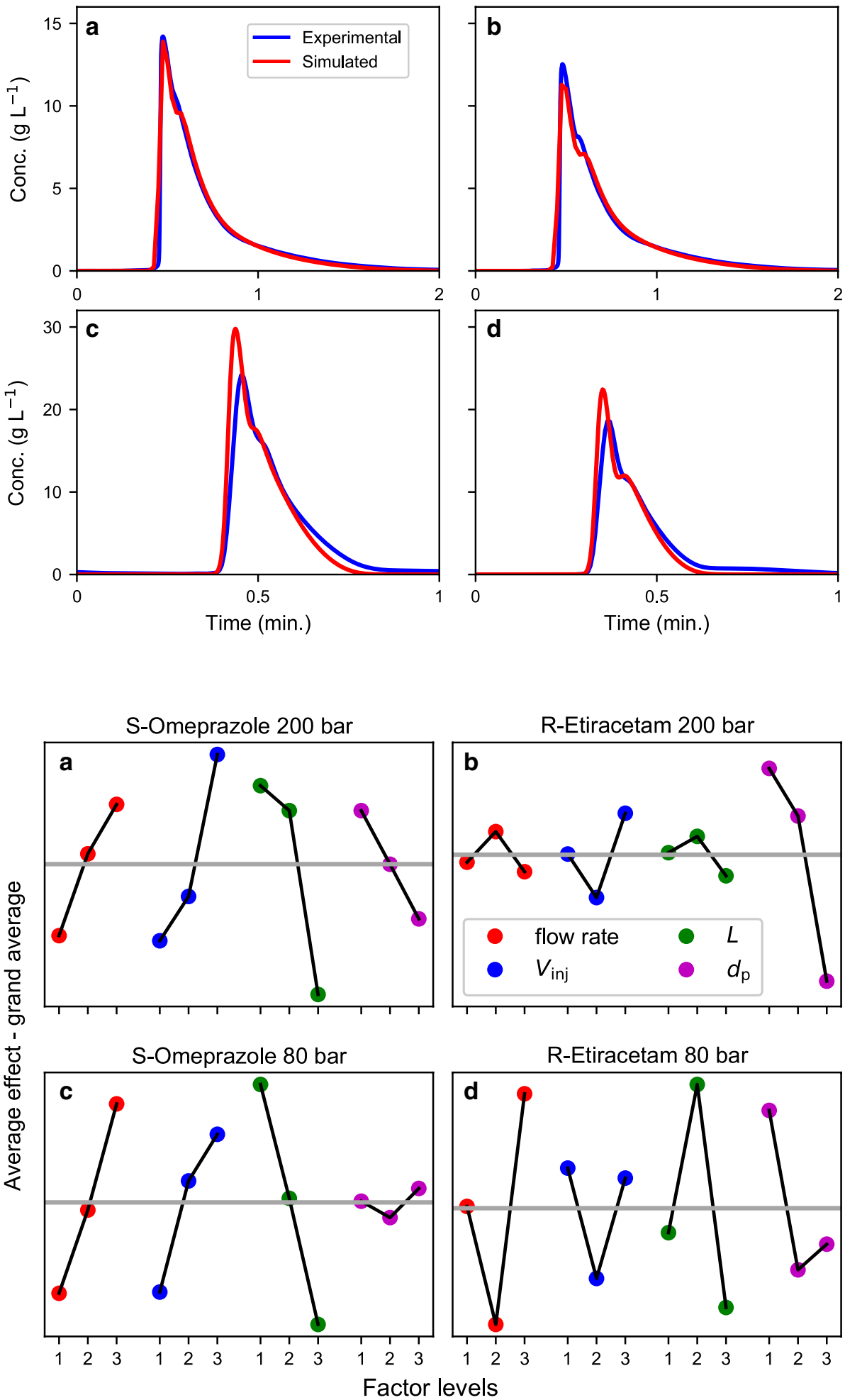
In the omeprazole case, roughly the same optimal conditions were predicted using both the numerical and Taguchi methods. At 80 bar, the Taguchi method suggested using smaller packing than did the numerical method. However, the average effects of stationary-phase packing particle diameter calculated using the Taguchi method were only slightly worse for $5-\mu \mathrm{m}$ than for $25-\mu \mathrm{m}$ particles (see Fig. 6c). Inspecting the productivity, in Table 1, the classical numerical method predicted much higher productivity for the 80 bar omeprazole separation for both enantiomers than did the Taguchi method. This is because Taguchi optimization was flow limited, i.e., operating at the maximum allowed flow rate. The maximum allowed flow rate in the Taguchi method was set to the maximum allowed flow rate for the longest column $(25 \mathrm{~cm})$ packed with the smallest stationary-phase packing particles $(5 \mu \mathrm{m})$. When the Taguchi method was flow limited, the maximum allowed flow rate could not be increased by decreasing the column length or increasing the packing material particle size, as can be done in classical numerical optimization. Therefore, the velocities obtained from Taguchi and from classical numerical optimization differed from each other.

In the etiracetam case, larger differences in optimal separation systems were found between the classical numerical and Taguchi methods than in the omeprazole case (Table 1). In this case, the Taguchi method suggested that longer columns packed with smaller packing particles should be used than did the numerical method. To explain these differences, first, we note that the separation was flow limited (except for $R$-etiracetam at $200 \mathrm{bar}$ ) in the Taguchi optimization. As a consequence, longer columns and smaller packing were found as optimum by the Taguchi method (see Fig. 6b, d). In the 200 bar case, the column length had very small impact on the optimum; see Fig. 6 b.

The Taguchi approach is very useful and successful, mainly in areas where optimal values of discrete decision variables, such as packing particle diameter and column length, are to be determined. The Taguchi method is easy to apply in such cases, but it may be difficult to establish appropriate Taguchi design spaces in more complex optimizations such as those presented here. To conclude, the Taguchi approach could be recommended for relatively simple optimization or pre-optimization to help in selecting column packing, etc. For accurate and complicated optimizations, however, classical numerical methods are still preferred.

\section{Conclusion}

In this study, we have considered, given a column of a certain diameter, what column length should be selected and what sized particles that the column should be packed with to achieve maximum productivity. We also compared advanced numerical optimization based on a mechanistic model with empirical optimization, i.e., the Taguchi method, with the latter being more readily available in the laboratory to the general chromatographer.

Both separation systems were numerically optimized to derive the optimal packing material particle size, column length, and flow rate. Maximum allowed backpressures of 80 and 200 bar were investigated. Preparative chromatography is often conducted using large-particle-diameter packing material and a column length of $25 \mathrm{~cm}$. Here, we demonstrated that, in the studied batch chromatography cases, shorter columns were more suitable when using packing materials with smaller stationary-phase particle sizes. In both investigated cases, a column length of $10 \mathrm{~cm}$ was found to be optimal. We also demonstrated that increasing the maximum allowed backpressure 2.5 times resulted in approximately 1.5 times greater productivity and a 0.6 times reduction in solvent consumption.

In this study, we also used the Taguchi method, a chemometric method that can work strictly from experimental data. The Taguchi method is easy to learn and use for the practical chromatographer. It is very suitable for quickly determining optimal discrete parameters or pre-estimating an objective function, as exemplified in this study by determining what column length and particle diameter should be used to achieve the highest productivity. In this study, we noted that short columns packed with smaller particles were preferable to longer columns packed with larger particles. The optimum process conditions were not exactly found, this is why we still recommend classical numerical optimization for the most accurate process optimization. However, this study clearly demonstrates that quite acceptable predictions can be achieved with less numerical effort.

Acknowledgements This work was supported by the Swedish Knowledge Foundation as part of the KKS SYNERGY project 2016 "BIOQC: Quality Control and Purification for New Biological Drugs" (grant number 20170059) and by the Swedish Research Council (VR) as part of the project "Fundamental Studies on Molecular Interactions aimed at Preparative Separations and Biospecific Measurements" (Grant number 2015-04627). We also thank the National Science Centre, Poland for support via Grant number 2015/18/M/ST8/00349. We are grateful to AstraZeneca and UCB Pharma for kindly giving us omeprazole and etiracetam, respectively, and to Kromasil/Akzo Nobel Pulp and Performance Chemicals $\mathrm{AB}$ for giving us the AmyCoat columns.

\section{Compliance with Ethical Standards}

Conflict of interest All authors declare that they have no conflict of interest.

Ethical approval This article does not contain any studies with human participants or animal performed by any of the authors.

Open Access This article is distributed under the terms of the Creative Commons Attribution 4.0 International License (http://creativeco 
mmons.org/licenses/by/4.0/), which permits unrestricted use, distribution, and reproduction in any medium, provided you give appropriate credit to the original author(s) and the source, provide a link to the Creative Commons license, and indicate if changes were made.

\section{References}

1. Bonilla JV, Srivatsa S (2011) Handbook of analysis of oligonucleotides and related products. CRC Press, Boca Raton

2. Zhang Q, Lv H, Wang L et al (2016) Recent methods for purification and structure determination of oligonucleotides. Int J Mol Sci 17:2134. https://doi.org/10.3390/ijms17122134

3. Cox GB, Antle PE, Snyder LR (1988) Preparative separation of peptide and protein samples by high-performance liquid chromatography with gradient elution: II. Experimental examples compared with theory. J Chromatogr 444:325-344

4. El Fallah MZ, Guiochon G (1992) Prediction of a protein band profile in preparative reversed-phase gradient elution chromatography. Biotechnol Bioeng 39:877-885. https://doi.org/10.1002/ bit. 260390810

5. Schmidt-Traub H (2005) Preparative chromatography: of fine chemicals and pharmaceutical agents. Wiley-VCH, New York

6. Cox GJ (2005) Preparative enantioselective chromatography, 1st edn. Blackwell, Ames

7. Åsberg D, Langborg Weinmann A, Leek T et al (2017) The importance of ion-pairing in peptide purification by reversed-phase liquid chromatography. J Chromatogr A 1496:80-91. https://doi. org/10.1016/j.chroma.2017.03.041

8. Andersson S, Nelander H, Öhlén K (2007) Preparative chiral chromatography and chiroptical characterization of enantiomers of omeprazole and related benzimidazoles. Chirality 19:706-715. https://doi.org/10.1002/chir.20375

9. Guiochon G, Shirazi DG, Felinger A, Katti AM (2006) Fundamentals of preparative and nonlinear chromatography, 2nd edn. Academic Press, Boston

10. Felinger A, Guiochon G (1996) Optimizing preparative separations at high recovery yield. J Chromatogr A 752:31-40

11. Åsberg D, Leśko M, Leek T et al (2017) Estimation of nonlinear adsorption isotherms in gradient elution RP-LC of peptides in the presence of an adsorbing additive. Chromatographia 80:961-966. https://doi.org/10.1007/s10337-017-3298-y

12. Enmark M, Arnell R, Forssén P et al (2011) A systematic investigation of algorithm impact in preparative chromatography with experimental verifications. J Chromatogr A 1218:662-672. https ://doi.org/10.1016/j.chroma.2010.11.029

13. Forssén P, Arnell R, Kaspereit M et al (2008) Effects of a strongly adsorbed additive on process performance in chiral preparative chromatography. J Chromatogr A 1212:89-97. https://doi. org/10.1016/j.chroma.2008.10.040

14. Forssén P, Arnell R, Fornstedt T (2009) A quest for the optimal additive in chiral preparative chromatography. J Chromatogr A 1216:4719-4727. https://doi.org/10.1016/j.chroma.2009.04.010

15. ICH Harmonised Tripartite Guideline (2017) Technical and Regulatory Considerations for Pharmaceutical Product Lifecycle Management Q12

16. Forssén P, Samuelsson J, Fornstedt T (2014) Relative importance of column and adsorption parameters on the productivity in preparative liquid chromatography II: investigation of separation systems with competitive Langmuir adsorption isotherms. J Chromatogr A 1347:72-79. https://doi.org/10.1016/j.chroma.2014.04.059

17. Enmark M, Samuelsson J, Forssén P, Fornstedt T (2012) Enantioseparation of omeprazole - effect of different packing particle size on productivity. J Chromatogr A 1240:123-131. https://doi. org/10.1016/j.chroma.2012.03.085
18. Forssén P, Samuelsson J, Fornstedt T (2013) Relative importance of column and adsorption parameters on the productivity in preparative liquid chromatography. I: investigation of a chiral separation system. J Chromatogr A 1299:58-63. https://doi org/10.1016/j.chroma.2013.05.031

19. Olbe L, Carlsson E, Lindberg P (2003) A proton-pump inhibitor expedition: the case histories of omeprazole and esomeprazole. Nat Rev Drug Discov 2:132-139. https://doi.org/10.1038/nrd10 10

20. Nicoud R-M (2014) The amazing ability of continuous chromatography to adapt to a moving environment. Ind Eng Chem Res 53:3755-3765. https://doi.org/10.1021/ie5005866

21. Antos D, Kaczmarski K, Wojciech P, Seidel-Morgenstern A (2003) Concentration dependence of lumped mass transfer coefficients: linear versus non-linear chromatography and isocratic versus gradient operation. J Chromatogr A 1006:61-76. https:// doi.org/10.1016/s0021-9673(03)00948-8

22. Introduction To Robust Design (Taguchi Method). https://www. isixsigma.com/methodology/robust-design-taguchi-method/intro duction-robust-design-taguchi-method/. Accessed 6 Dec 2016

23. Kaczmarski K, Antos D, Sajonz H et al (2001) Comparative modeling of breakthrough curves of bovine serum albumin in anionexchange chromatography. J Chromatogr A 925:1-17

24. Kaczmarski K, Mazzotti M, Storti G, Mobidelli M (1997) Modeling fixed-bed adsorption columns through orthogonal collocations on moving finite elements. Comput Chem Eng 21:641-660. https://doi.org/10.1016/s0098-1354(96)00300-6

25. Kaczmarski K, Zhou D, Gubernak M, Guiochon G (2003) Equivalent models of indanol isomers adsorption on cellulose tribenzoate. Biotechnol Prog 19:455-463. https://doi.org/10.1021/bp020 $117 \mathrm{~m}$

26. Wilson EJ, Geankoplis CJ (1966) Liquid mass transfer at very low reynolds numbers in packed beds. Ind Eng Chem Fundam 5:9-14. https://doi.org/10.1021/i160017a002

27. Wilke CR, Chang P (1955) Correlation of diffusion coefficients in dilute solutions. AIChE J 1:264-270. https://doi.org/10.1002/ aic.690010222

28. Forssén P, Arnell R, Fornstedt T (2006) An improved algorithm for solving inverse problems in liquid chromatography. Comput Chem Eng 30:1381-1391. https://doi.org/10.1016/j.compchemen g.2006.03.004

29. Forssén P, Fornstedt T (2015) A model free method for estimation of complicated adsorption isotherms in liquid chromatography. J Chromatogr A 1409:108-115. https://doi.org/10.1016/j.chrom a.2015.07.030

30. Press WH, Teukolsky SA, Vetterling WT, Flannery BP (2002) Numerical recipies in C. Cambridge University Press, Cambridge

31. Kaczmarski K, Antos D (2006) Use of simulated annealing for optimization of chromatographic separations. Acta Chromatogr 17:20-45

32. Wang S, Guo M, Cong J, Li S (2013) Facile optimization for chromatographic separation of liquiritin and liquiritigenin. J Chromatogr A 1282:167-171. https://doi.org/10.1016/j.chrom a.2013.01.075

33. Futagawa T, Canvat JP, Cavoy E, et al (2000) By optical resolution of a racemic mixture of alpha-ethyl-2-oxo-1-pyrrolidine acetamide by chromatography using silica gel supporting amylose tris(3,5-dimethylphenylcarbamate) as a packing material. US 6107492 A

34. Gubernak M, Zapala W, Tyrpien K, Kaczmarski K (2005) Analysis of amylbenzene adsorption equilibria on different reversedphase HPLC. J Chromatogr Sci 42:457-463 